\title{
Fundamental and practical aspects concerning the characterization of smart textiles
}

\author{
Gianina Broasca1, Gabriela Borcia $^{2}$, Nicoleta Dumitrascu ${ }^{2}$, Marius Cioca $^{3}$, Diana Coman $^{3}$, \\ Nouredine Ourfelli ${ }^{4}$, and Narcisa Vrînceanu ${ }^{3, *}$ \\ 1 "Gheorghe Asachi” Technical University of Iaşi, 53 Mangeron Street, Romania \\ 2 "Alexandru Ioan Cuza" University of Iaşi, 21 Carol I Street, Romania \\ ${ }^{3}$ Lucian Blaga University of Sibiu, 4 Emil Cioran St, Romania \\ 4 Université de Tunis El Manar, Laboratoire de Biophysique et Technologies Médicales; \\ Abdulrahman Alfaisal University, Department of Chemistry, College of Science, Saudi Arabia
}

\begin{abstract}
The research aims towards a novel methodology of $\mathrm{ZnO}$ impregnation onto polyamide supports, in order to attain a special inorganic-organic hybrid polymer material, with increased UV-barrier attributes and high hydrophobicity. The experimental approach employed $\mathrm{ZnO}$ micro particles powder. Solutions with different concentrations of $\mathrm{ZnO}$ powder dispersed in methanol are prepared as anti-UV finishing agent and applied onto polyamide fabric. The responsive behaviour of this complex polymer network to UV irradiation, the photo protective performance and its time stability, also the hydrophobic character are assessed by different characterization techniques. The proposed method has advantages, like: easy standardization, at lower production cost, resulting a high homogeneity and dispersion of $\mathrm{ZnO}$ micro particles into the textile polymeric network. The covered polymeric supports show better photo protective response, higher hydrophobicity and control on the surface charge induced by irradiation. This method granted enhanced quality, comfort and long term stability of the intelligent/smart garments.
\end{abstract}

\section{Introduction}

The textile area intents to design fabrics accommodated to strict surroundings conditions, as to promote the human physiological comfort, by facile and reduced cost finishing procedures. Commonly, the fabrics sector needs relevant procedures from the processing of raw materials to the distinct finished items [1-3].

The attributes of garments are functions of the processing technique. In this regard, the nanotechnologies polarized much significance in the textiles area, granting to realize multifunctional materials with attractive properties, controlled in the $0.1-100 \mathrm{~nm}$ range. Recently, the embodiment of metal particles (e.g., zinc, silver, gold, platinum, etc.) was analysed, in order to attain novel textiles [4]. For instance, the silver nanoparticles have been embodied within different polymeric fibers belonging to various fabrics, primarily to provide added value features, like: stain-repellence, bactericidal properties, or blocking of

${ }^{*}$ Corresponding author: vrinceanu.narcisai@ulbsibiu.ro 
smelly and odorous vapours [4]. Nevertheless, these metals occur in the form of nanoparticles, meaning that these can be destructive to health if absorbed by the human body [4]. Such issues need extended research efforts for back-up covering/incorporating polymeric supports.

The barrier against the UV radiation is a practical demand for the safety of garments designed for particular applications, as extended sun exposure during outdoor activities. Due to their unique properties, like: electrical, photocatalytic, optical, dermatological and antibacterial, zinc oxide $(\mathrm{ZnO})$ powders are widely used semiconductor materials, having numerous structures. Furthermore, for medical applications $\mathrm{ZnO}$ is bio-safe and biocompatible. $\mathrm{ZnO}$ is experienced as a UV-blocking system, mainly in the UV-A region. In order to apply $\mathrm{ZnO}$ powder as UV-absorbing system, it has to be assimilated into different matrices, by means of diverse approaches developed to improve the stability of $\mathrm{ZnO}$ in different dispersions media and to block particles clustering.

Considering all these, a novel methodology based on $\mathrm{ZnO}$ impregnation of polyamide supports in order to engineer an inorganic-organic hybrid polymer network, with augmented UV-barrier attributes and high hydrophobicity was nominated. The experimental component consists of employing $\mathrm{ZnO}$ microparticles powder. As anti-UV finishing agent different concentrations of solutions with of $\mathrm{ZnO}$ powder dispersed in methanol were prepared and applied onto polyamide fabric. Different characterization methods were used to evaluate the responsive behaviour of this complex polymer matrix to UV irradiation, such as: the photoprotective work, long term stability, the hydrophobic character. As advantages of this solution we can mention:

- Easy standardization,

- Lower production cost,

- High homogeneity and dispersion of $\mathrm{ZnO}$ microparticles into the textile polymeric network.

\section{Experimental}

\subsection{Materials and preparation}

Woven polyamide fabrics $\left(83.5 \mathrm{~g} / \mathrm{m}^{2}\right)$ were identified and prepared for immersion in the form of $3 \mathrm{~cm}-7 \mathrm{~cm}$ strips. The micrometer-sized $\mathrm{ZnO}$ powder was supplied by Ensait Laboratory, France. Laser diffraction was employed to perform the particle-size distribution, showing that $75 \%$ of the $\mathrm{ZnO}$ powder consists of $1.7 \mu \mathrm{m}$ size particles. The solutions are based of $\mathrm{ZnO}$ powder dispersed in methanol (99.8\%), at different concentrations: $1 \%, 3 \%, 5 \%$ and $7 \%$. The magnetic stirring was used for 15 minutes, in order to obtain the solutions, adding 4 drops of Vitexol (BASF) to avert foaming and $65 \mathrm{~g} / 1$ Apretan (Clariant) to provide the fixation of $\mathrm{ZnO}$ to fibers.

The studied samples were immersed into $\mathrm{ZnO}$ solutions by padding, on a Wemer Mathis AG Laboratory machine, followed by wet-picking, pressing the textile between hydroextraction cylinders, in order to eliminate a part of liquid/paste from the polymeric surface. The samples are weighted before and after impregnation. The immersed specimens were exposed in a Vetter machine for drying, for 3 minutes, at $110^{\circ} \mathrm{C}$. The mixture is then fastened on the fabric, heated at $150{ }^{\circ} \mathrm{C}$ for 3 minutes, by thermal induced crosslinking [5]. 


\subsection{Characterization methods}

Scanning electron microscope (SEM) images of the samples are obtained with a Quanta 200 3D Dual Beam type microscope (FEI Holland), coupled to an energy-dispersive X-ray spectroscopy (EDS) analysis system (EDAX - AMETEK Holland) equipped with a SDD (silicon drift detector) type detector. Taking into account the sample type, the analyses are performed using Low Vacuum working mode, allowing the probes tested in their initial state, without a previous metallization. Both for the acquisition of secondary electrons images and EDS elemental chemical analysis, LFD (Large Field Detector) detector is used, running at $60 \mathrm{~Pa}$ pressure and $30 \mathrm{kV}$ voltage.

The wicking of the polymeric support is assessed by contact angle measurement. The sessile drop technique was used in order to obtain the contact angles, under controlled conditions of room temperature and humidity. An automated system stored the drop images, via a Canon A85 camera, with PC-based control, acquisition and data processing.

Ten measures on the imaged sessile liquid drop profile, for $1 \mu 1$ drop size were performed. By averaging these values, the values of the static contact angle were obtained. As test liquid distilled water was employed. The adhesion activity of water on the surface, meaning wicking, is calculated as follows:

$$
W_{a}=\gamma_{l v}(1+\cos \theta)
$$

Where $W_{a}$ is the contact angle and $Y_{l v}$ is the surface tension.

\section{Results and discussion}

Figure 1 displays SEM microphotographs of polyamide supports covered with $\mathrm{ZnO}$. The surface belonging to the reference polyamide fabric is smooth (Figure 1a), exposing only tiny detached particles, like impurities.

Different concentrations of $\mathrm{ZnO}$ solutions lead to different layer covering the polyamide samples, having different morphology based on the increasing $\mathrm{ZnO}$ concentration of the finishing agent.

For the sample treated with $1 \% \mathrm{ZnO}$, a thin film, with lamellar morphology and good uniformity, was obtained (Figure 1b). The thickness of this layer increments with an increased $\mathrm{ZnO}$ concentration, displaying a uniform coating of the supports for $3-5 \% \mathrm{ZnO}$ (Figure 1c).

In case of higher concentration in zinc oxide, a lower dispersion of the microparticles was noticed, along with the visible clusters leading to non-uniform covering of the supports (Figure 1d). Consequently, an uniform covering of fibers is achieved due to the best dispersion of the particles, correlated to $\mathrm{ZnO}$ concentration lower than $5 \%$.

The covering of the supports is sustained by EDS measurement, where the $\mathrm{Zn}$ quantity on the specimens displays correlation to the $\mathrm{ZnO}$ concentration of the solutions. It was demonstrated that the content is the highest for the $7 \% \mathrm{ZnO}$ sample.

The wicking of the polymeric support surface evaluates the material behaviour in presence of water or humid environment. The values are gathered in Table 1. Moreover, rerun measurement demonstrates that the contact angle is not altered by macroscopic nonuniformity of the woven materials, since all liquid drops deposited onto the surface show regular, symmetric profile. 

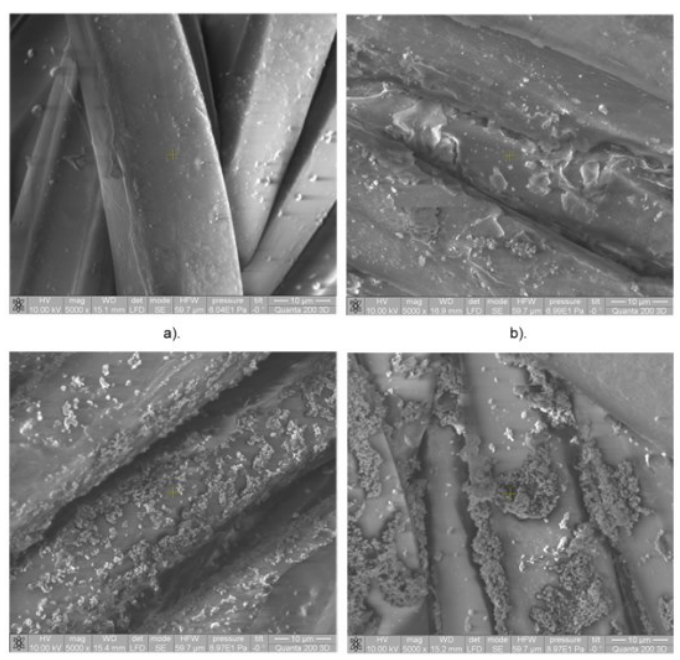

c).

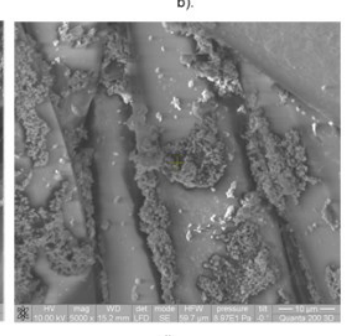

d).

Fig. 1. SEM photos of polyamide supports covered with $\mathrm{ZnO}$ : a) reference, b) $1 \% \mathrm{ZnO}$, c) $5 \% \mathrm{ZnO}$, d) $7 \% \mathrm{ZnO}$.

Generally speaking, the polyamide fabric is hydrophobic, having a water contact angle higher than $90^{\circ}$. In this case the surface is not wet. In addition, the polymeric support does not absorb the water, because the drops deposited onto the surface vanish after long intervals, by evaporation.

The padding with $\mathrm{ZnO}$ pointed out, in a visible manner, a higher hydrophobicity, as the augmentation of contact angle. This aspect can be regarded as a diminution of the adhesion activity, with an average $50 \%$. This fact certifies that the polymeric supports are covered with an oxide layer, which is more hydrophobic than the polymer itself.

Another interesting fact is the lowest wicking noticed for $3 \% \mathrm{ZnO}$ impregnated polymeric support, while incremented $\mathrm{ZnO}$ concentration conducts to higher wicking, i.e. reversal of the surface attributes. Between the 3\% and 7\% $\mathrm{ZnO}$ samples a difference of $68 \%$ has been calculated, which is relevant.

This response can be regarded as a function of a better dispersion of $\mathrm{ZnO}$ particles in the $3 \%$ specimen, which is more diluted, granting more homogenous covering of textile supports, and more hydrophobic attribute due to oxide film. In case of samples coated with higher oxide concentration, the cluster of $\mathrm{ZnO}$ particles leads to a low levelness, consequently the fibers are irregularly covered with films of different thickness.

Table 1. Contact angle and adhesion responsive attitude of water on polyamide supports covered with $\mathrm{ZnO}$, before and after $\mathrm{UV}$ irradiation.

\begin{tabular}{|c|c|c|c|c|}
\hline \multirow{2}{*}{ Samples } & \multicolumn{2}{|c|}{ before UV irradiation } & \multicolumn{2}{c|}{ after UV irradiation } \\
\cline { 2 - 5 } & $\theta\left({ }^{\circ}\right)$ & $W_{a}\left(\mathbf{m J} / \mathbf{m}^{2}\right)$ & $\theta\left(^{\circ}\right)$ & $W_{a}\left(\mathbf{m J} / \mathbf{m}^{2}\right)$ \\
\hline polyamide & $92^{\circ}$ & 67 & $88^{\circ}$ & 75 \\
\hline polyamide $+1 \% \mathrm{ZnO}$ & $120^{\circ}$ & 32 & $115^{\circ}$ & 30 \\
\hline polyamide $+3 \% \mathrm{ZnO}$ & $131^{\circ}$ & 20 & $135^{\circ}$ & 27 \\
\hline polyamide $+5 \% \mathrm{ZnO}$ & $129^{\circ}$ & 26 & $124^{\circ}$ & 32 \\
\hline polyamide $+7 \% \mathrm{ZnO}$ & $113^{\circ}$ & 33 & $116^{\circ}$ & 48 \\
\hline
\end{tabular}


The UV irradiation of the specimens leads, as expected, to the decreasing of the contact angle. This decreasing is noticeable for the polyamide fabric, in terms of the surface chemistry modification of polymers under exposure to energy sources. The modification is important less for the supports covered with $\mathrm{ZnO}$. In this manner the UV-barrier function of the oxide layer was stressed. It is noteworthy to mention that all the studied $\mathrm{ZnO}$ covered probes show a relevant hydrophobicity after UV irradiation. The 7\% $\mathrm{ZnO}$ sample showed the highest level of modification. The synergic effect provided by the better dispersion of the microparticles $(3-5 \% \mathrm{ZnO}$ solutions) and the good levelness of the surface layer, showed an improved UV-protection.

\section{Conclusion}

A novel methodology is oriented toward the impregnation of $\mathrm{ZnO}$ powder in a polymeric matrix, applicable, in order to obtain textiles with a multifunctional performance: UV barrier, high hydrophobicity.

The method provides a homogenous dispersion of $\mathrm{ZnO}$ particles through the polymeric support. New attributes like higher photoprotection, higher hydrophobicity and assessment on the irradiated surface.

This experimental approach maintains the mechanical features of the material unmodified, providing an improved quality, comfort and long term stability of the clothing.

\section{References}

1. Y.W.H. Wong, C.W.M. Yuen, M.Y.S. Leung, S.K.A. Ku, H.L.I. Lam, Autex Research Journal, 6 (2006)

2. K.L. Hatch, U. Osterwalder, Dermatologic Clinics, 24 (2006)

3. J.M. Menter, K.L. Hatch, in: P. Elsner, K. Hatch, W. Wigger (Eds.), Textiles and the Skin, Current Problems in Dermatology, 31, Karger, Basel (2003)

4. C.H. Xue, W. Yin, S.T. Jia, J.Z. Ma, Nanotechnology, 22 (2011)

5. G. Broasca, G. Borcia, G. Dumitrascu, N. Vrinceanu, Applied Suface Science, 279 (2013) 